\title{
Surface structure of cubic aluminum oxide
}

\author{
Luis Javier Alvarez and Luis Eduardo León \\ Dirección General de Servicios de Cómputo Académico, Universidad Nacional Autónoma de México, Circuito Exterior, \\ Cuidad Universitaria, 04510 México, Distrito Federal, Mexico \\ Javier Fernández Sanz ${ }^{*}$ \\ Departamento de Química Física, Facultad de Química, Universidad de Sevilla, E-41012 Sevilla, Spain \\ María José Capitán and José Antonio Odriozola \\ Departamento de Química Inorgánica e Instituto de Ciencia de Materiales, \\ Universidad de Sevilla-Consejo Superior de Investigaciones Científicas, E-41012 Sevilla, Spain
}

(Received 22 February 1994)

\begin{abstract}
Molecular-dynamics simulations using a Pauling type pairwise potential have been carried out in order to study the structure of the surface of $\gamma-\mathrm{Al}_{2} \mathrm{O}_{3}$. Starting from an ideal (100) face, a reconstruction process occurs in which the unbalanced coordination of surface ions tends to fulfill their coordination capabilities. Oxygen ions come to the surface and pore formation with a concomitant increase of apparent volume takes place. The structure of the surface of the resulting material corresponds to a porous amorphouslike phase in which only short-range order is present. Analysis of the radial distribution function agrees with experimental x-ray-diffraction data.
\end{abstract}

\section{INTRODUCTION}

Since the pioneering work by Peri $^{1}$ the surface of $\gamma$ $\mathrm{Al}_{2} \mathrm{O}_{3}$ has been the object of considerable interest because of its importance as catalyst and as a catalyst support. Most of the surface models are idealized views of the catalyst surface. In Peri's model the (100) surface of $\mathrm{NaCl}$ type structure is considered when it is well known that $\gamma-\mathrm{Al}_{2} \mathrm{O}_{3}$ has a defective spinel structure. Later models considered the actual structure of the solid but with an excess of aluminum cations to solve the problem of the random occupation of tetrahedral and octahedral sites. ${ }^{2,3}$

In all these surface models the coordination of cations at the surface is considered to be tetrahedral and octahedral. However, this assumption disagrees with data reported recently by Chen, Davis, and Fripiat, ${ }^{4}$ who from magic angle spinning $\mathrm{NMR}^{27} \mathrm{Al}$ spectroscopy found the existence of five-coordinated aluminum atoms. Recently, we reported molecular-dynamics simulations on $\gamma-\mathrm{Al}_{2} \mathrm{O}_{3}$ in which the actual stoichiometry was taken into account. $^{5}$ Also, from cleavage fracture planes of the bulk, idealized faces according to the most usually accepted exposed surfaces were reported. ${ }^{6}$ In our work, we showed that five-coordinated aluminum ions occur both in the bulk and at the surface. Nevertheless, none of these efforts to understand the structure of the $\gamma-\mathrm{Al}_{2} \mathrm{O}_{3}$ considered the reconstruction of the outer layers of the solid.

Surface reconstruction of metals is a well-described phenomenon; ${ }^{7}$ however, in the case of nonconducting solids such as metal oxides, very little has been achieved due to the experimental limitations regarding the obtainment of LEED patterns, and only reconstruction of few surfaces such as $\mathrm{ZnO}, \mathrm{TiO}_{2}$, and $\alpha-\mathrm{Al}_{2} \mathrm{O}_{3}$ have been described. ${ }^{8}$ The experimental study of the reconstruction of $\gamma-\mathrm{Al}_{2} \mathrm{O}_{3}$ has the additional limitation of the crystal size, although experimental data based on adsorption of probe molecules suggest that a surface reconstruction indeed takes place. $^{3}$

The theoretical and experimental limitations mentioned above make the study of the structure of the surface of metal oxides in general and particular of $\gamma-\mathrm{Al}_{2} \mathrm{O}_{3}$ suitable for numerical simulation. In this work we report molecular-dynamics simulations of the surface layers of $\gamma-\mathrm{Al}_{2} \mathrm{O}_{3}$ in order to study their structure and surface reconstruction.

\section{COMPUTATIONAL PROCEDURE}

The interaction potential used in our simulations is the same as the one used in previous works on alumina. This potential was shown to give excellent results concerning both the structure of the bulk, through the calculation of radial distribution functions, and the energetics of the system, predicting heat of formation and vibrational spectra in agreement with experimental data. ${ }^{5}$ The potential is of Pauling type and has a Coulombic term plus a steric repulsion given by

$$
V\left(r_{i j}\right)=\frac{q_{i} q_{j} e^{2}}{r_{i j}}\left[1-\operatorname{sgn}\left(q_{i} q_{j}\right)\left[\frac{\sigma_{i}+\sigma_{j}}{r_{i j}}\right)^{n}\right],
$$

where $r_{i j}$ is the interionic distance, $q_{i}$ are the effective charges, and $\sigma_{i}$ are the ionic radii. The exponent $n$ was taken to be 9 according to Adams and McDonald. ${ }^{9}$ The effective charges of aluminum and oxygen were calculated from $a b$ initio self-consistent-field-molecular orbital calculations carried out on a series of small clusters of $\mathrm{Al}(\mathrm{OH})_{x}$ using HONDO-8.4 program. ${ }^{10}$ In these clusters, $x$ ranges from 2 to 6 , and thus effective charges are the weighted average of the most common coordination po- 
lyhedra of cubic alumina. The oxygen radius was that proposed by Shannon and Prewitt, ${ }^{11}$ whereas aluminum radius was chosen in such way as to minimize the differences between tetrahedral and octahedral coordinations. $^{12}$

The system consisted of 1440 particles in an originally cubic configuration of 27 unit cells at a density of 3.66 $\mathrm{g} \mathrm{cm}^{-3}$. Periodic boundary conditions were taken in the $x$ and $y$ directions. The long-range Coulombic interaction was handled with the Ewald summation method and a time step of $10^{-3} \mathrm{ps}$ was used. The initial configuration was taken from a previous work, ${ }^{5}$ where the system was equilibrated at $300 \mathrm{~K}$ for $5.5 \mathrm{ps}$ in the bulk. After removing the boundary conditions in the $z$ direction only the upper 720 particles were allowed to move, leaving the system free to evolve for 10 ps rescaling velocities when temperature increased above $300 \mathrm{~K}$. After this equilibration process a 5 ps production run was performed in which temperature variations were of less than $5^{\circ}$ and energy fluctuations were of less than $0.1 \%$. Calculations were performed using SIMULA $\operatorname{code}^{13}$ running on the Cray YMP/464 supercomputer of the Universidad $\mathrm{Na}$ cional Autónoma de México computing center.

\section{RESULTS AND DISCUSSION}

The removal of the boundary conditions in the $z$ direction generates coordination defects of surface ions ${ }^{4}$ which, in turn, give way to uncompensated interaction potential energy. The system tends to eliminate defects and to compensate the interaction potential energy by altering both position and coordination of atoms at the outermost layers. Along with this compensation there is a pore formation process which results in an increase of the apparent volume. After the equilibration stage the local structural characteristics such as bond distances and coordination numbers of the particles are preserved, keeping only short-range order. Figure 1 shows a

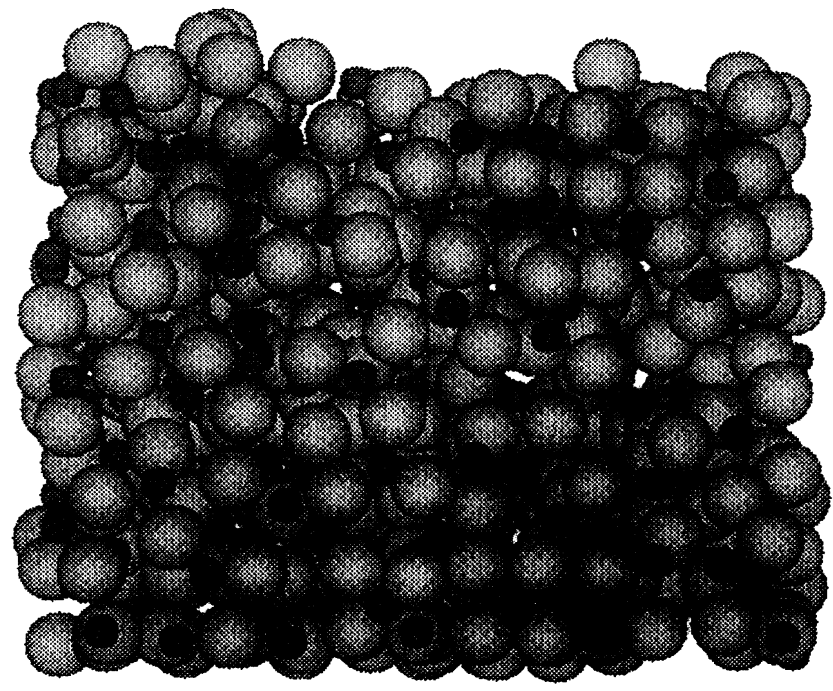

FIG. 1. Snapshot of the moving particles of the system after 15 ps simulation time. snapshot of the moving particles of the system after the 15 ps simulation run where some pores can be observed.

\section{A. Porosity}

In order to give an insight into the existence and distribution of pores in the surface, we have calculated the apparent density profile along the $z$ direction shown in Fig. 2. The plotted values were obtained calculating the density of a set of horizontal slabs $3 \AA$ thick. At depths larger than half the original box size $(11.8 \AA)$ the density values lie around the density of the original system in the bulk of $3.66 \mathrm{~g} \mathrm{~cm}^{-3}$, and the values at lower depths vary around $2.3 \mathrm{~g} \mathrm{~cm}^{-3}$. The variations of density in this region are due to the presence of large pores in the structure, and the lower oscillations around the mean value with respect to a crystalline structure are due to the nearly homogeneous distribution of pores. The calculated pore volume yields $0.07 \mathrm{~cm}^{3} \mathrm{~g}^{-1}$, which is within the limits of the expected range of micropore volume reported for $\gamma-\mathrm{Al}_{2} \mathrm{O}_{3}$ between 0.04 and $1.3 \mathrm{~cm}^{3} \mathrm{~g}^{-1}$. ${ }^{14}$ This value was obtained on the basis of fitting spheres of radii larger than $2.0 \AA$ to avoid accounting for interstitial volume. Assuming slit-shaped pores, as experimentally observed, the minimum distance between the alumina lamellae would be $4.0 \AA$. The average width being $5.1 \AA$ with a maximum of up to $8.2 \AA$. Figure 3 shows a view of the surface layers up to a depth of $5 \AA$ where cavities of different sizes can be observed. The largest one near the upper left corner is $10.5 \AA$ long, $4.0 \AA$ wide and its depth was estimated to be around $8 \AA$. From our simulations it can be stated that the micropore volume is not only due to empty octahedral sites as has been suggested, but also and more important, to the existence of genuine pores in an amorphous superficial phase.

In Fig. 3, where a large elliptical pore is shown, it can

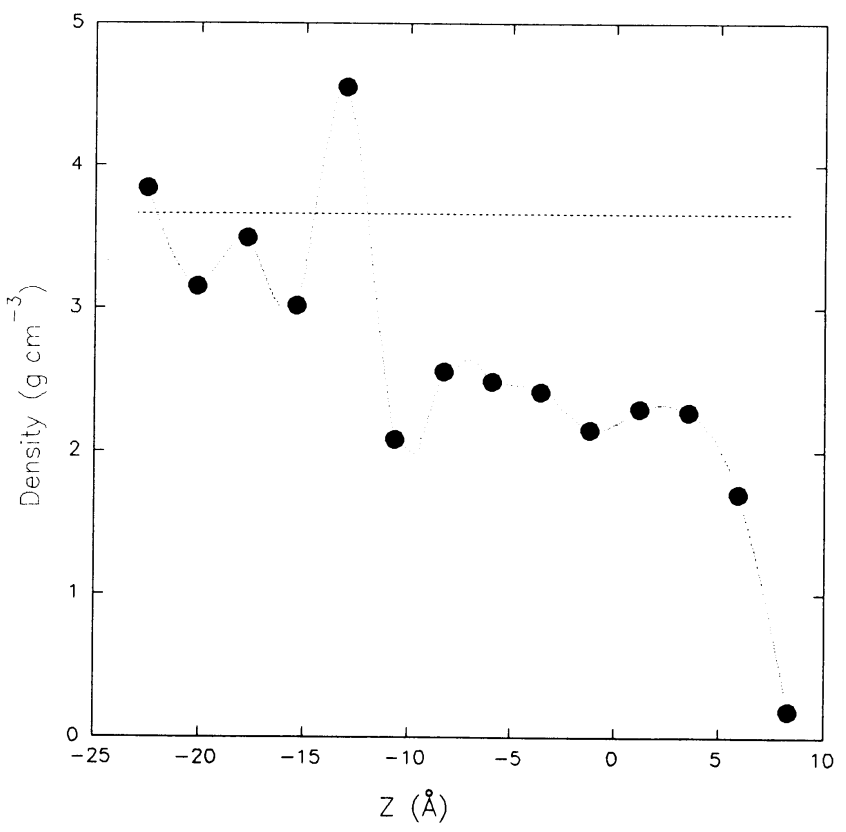

FIG. 2. Apparent density profile along the $z$ direction. 


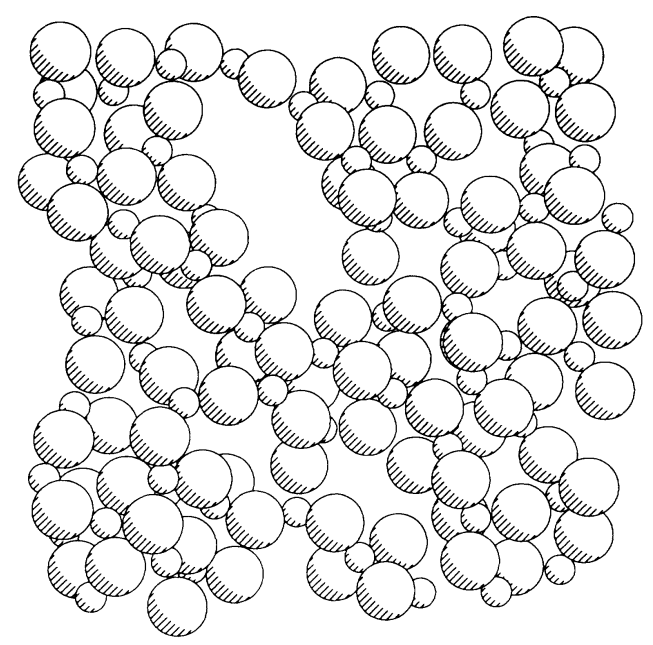

FIG. 3. View of the surface layers up to a depth of $5 \AA$. Cavities of different size can be observed.

be seen that its main axis is parallel to the (110) crystallographic direction and smaller pores are located along (110) and (110) directions. Figure 4 shows a slab of the whole system $3 \AA$ thick, and parallel to the $z-y$ plane. In the region where particles are allowed to move, there are pores of different size and shape, however, their location and orientation is along planes parallel or perpendicular to the (110) direction. This same feature can be observed at the surface where the uppermost layer has a jigsaw shape also oriented along these planes. In summary, in our simulations, starting from a surface exposing the (100) surface plane there is a reconstruction process at the end of which the surfaces exposed both at the gas solid interface and within pore systems are parallel or perpendicular to the (110) plane. This result agrees with experimental work in which the (110) plane has been found to be the more likely exposed., ${ }^{3,15}$ One additional question concerns the conditions under which a reconstruction of the type described here can occur in real sys-

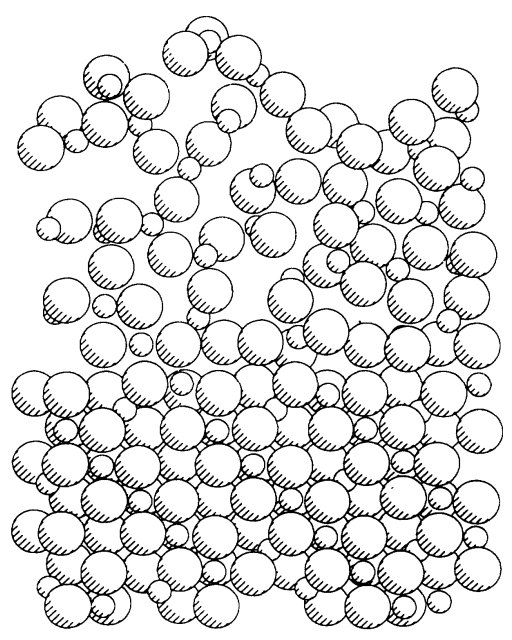

FIG. 4. Slab of the whole system $3 \AA$ thick, parallel to the $z-y$ plane. tems. Taking into account the experimental conditions under which the synthesis of cubic aluminas is performed, namely high temperature, they would never expose a surface of the (100) type, and in fact, a reconstruction such as that reported in this work would never occur.

\section{B. Radial distribution functions}

The radial distribution functions, $g(r)$, of the moving particles have been calculated in order to compare them with the ones calculated from experimental x-raydiffraction data (XRD). Figure 5 shows the partial $g(r)$ for the pairs Al-Al, Al-O, and O-O, along with the positions of maxima obtained from the experimental data up to $6 \AA$. The shift of positions of maxima of the calculated $g(r)$ with respect to the XRD results is at most $0.1 \AA$; therefore, using the partial radial distribution functions it is possible to identify their contributions to the total $g(r)$ shown in Fig. 6. The structure of radial distribution functions indicates the existence of short-range order in an amorphouslike phase, long-range order being hardly observable. This is due to the porous structure of the system in which although there is certain order, and a tendency to expose a plane similar to the (110) plane of the ideal crystal, the long-range order is not present. The $\mathrm{x}$ ray-diffraction pattern of $\gamma-\mathrm{Al}_{2} \mathrm{O}_{3}$ presents broad and poorly resolved peaks indicating an amorphous or a very

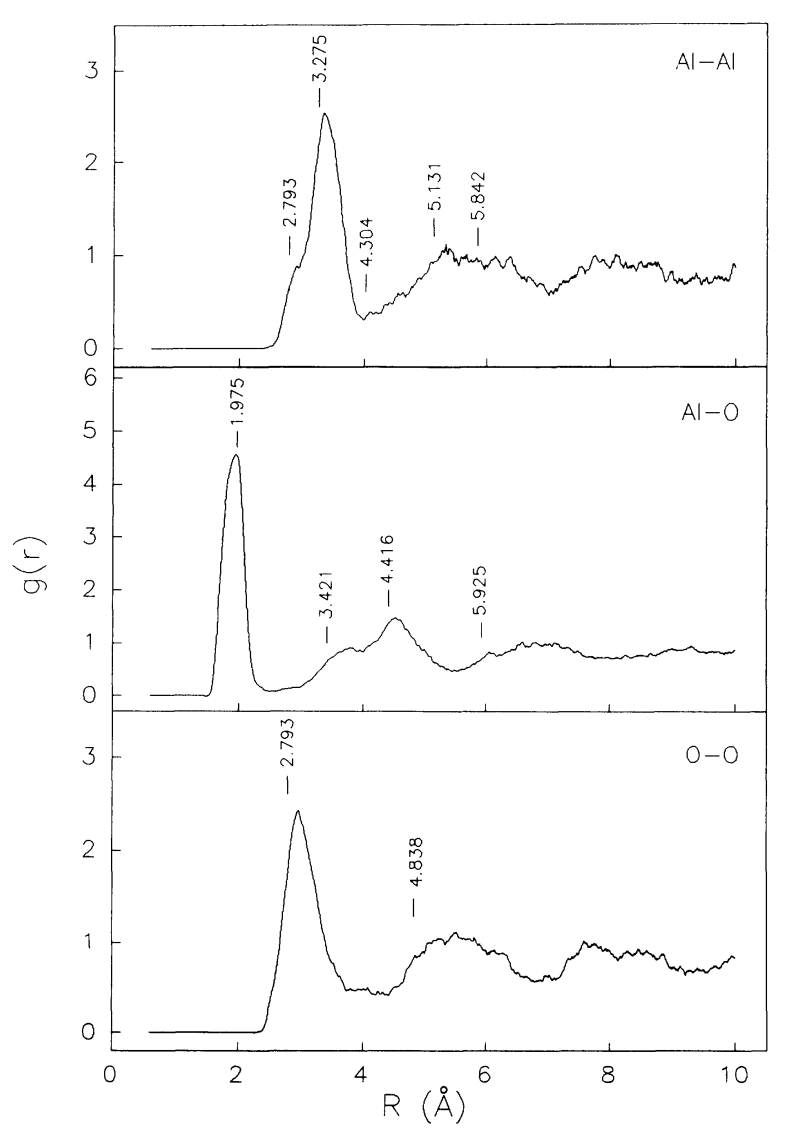

FIG. 5. Partial $g(r)$ for the pairs Al-Al, Al-O, and O-O along with the positions of maxima obtained from experimental data. 


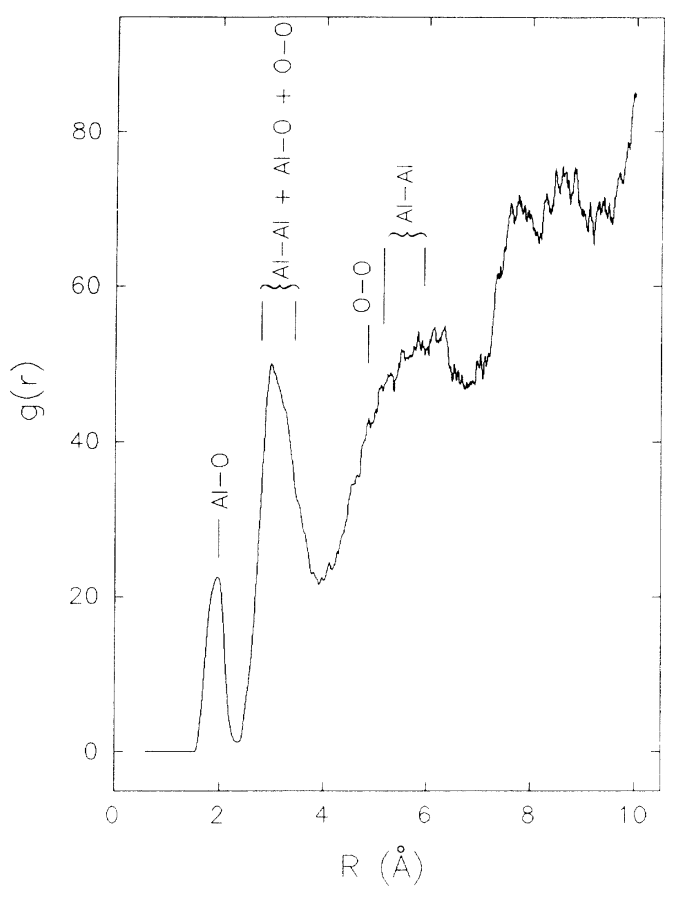

FIG. 6. Contributions of partial radial distribution functions to the total $g(r)$.

poorly crystallized material. Figure 7 shows experimental XRD patterns of three samples of $\gamma-\mathrm{Al}_{2} \mathrm{O}_{3}$ of different origin $^{16}$ and, for the sake of comparison, the XRD pattern of a sample of $\alpha-\mathrm{Al}_{2} \mathrm{O}_{3}$ obtained by calcining at 1473 $\mathrm{K} \gamma-\mathrm{Al}_{2} \mathrm{O}_{3}$ samples in air. As it is well known the inten-

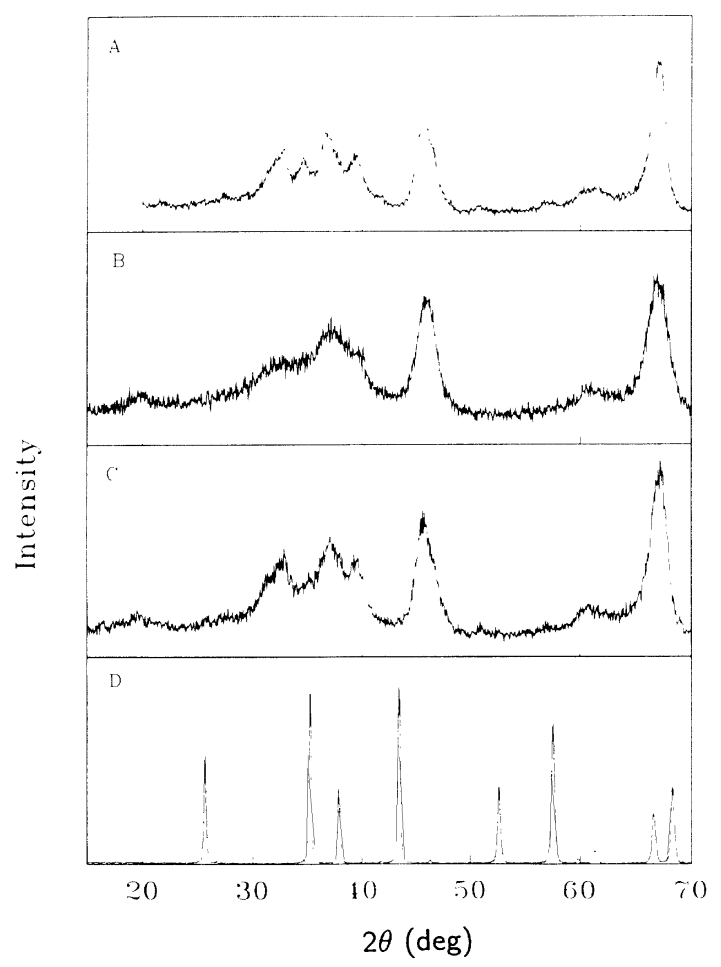

FIG. 7. Experimental XRD patterns of $\gamma$ and $\alpha-\mathrm{Al}_{2} \mathrm{O}_{3}$ obtained under different experimental conditions.

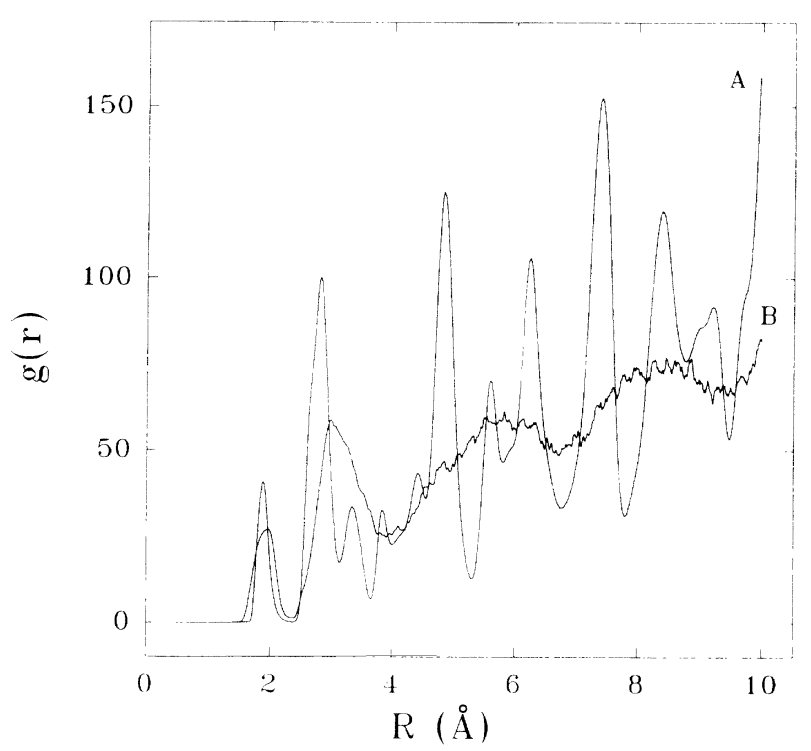

FIG. 8. Total $g(r)$ of the ideal spinel $(A)$ and of the resulting solid after eliminating boundary conditions in the $z$ direction $(B)$.

sity ratio and resolution of peaks in the XRD spectra is a function of the preparation method. The poorly resolved diffractograms correspond to samples with higher specific surface areas [(a) $70 \mathrm{~m}^{2} \mathrm{~g}^{-1}$; (b) $167 \mathrm{~m}^{2} \mathrm{~g}^{-1}$; (c) 127 $\mathrm{m}^{2} \mathrm{~g}^{-1}$; (d) $5 \mathrm{~m}^{2} \mathrm{~g}^{-1}$, and consequently with larger pore volume. On the basis of the above discussion the structure of catalytically active $\gamma-\mathrm{Al}_{2} \mathrm{O}_{3}$ may be understood as an amorphous material having short-range order in some way resembling the disordered spinel structure previously reported. ${ }^{4}$ This idea is illustrated in Fig. 8 where the total $g(r)$ of the ideal spinel structure, curve $A$, and that of the resulting solid after eliminating the boundary condition in the $z$ direction, curve $B$, are shown.

Table I shows the percentages of the observed coordination polyhedra for aluminum and oxygen in the ideal spinel and the simulated surface. The relative proportion of tetrahedral polyhedra of aluminum at the surface increases with respect to the ideal spinel structure at the expense of the octahedrally coordinated ones. First of all, the truncation of the bulk produces a decrease of coordi-

TABLE I. Observed percentages of coordination polyhedra for aluminum and oxygen in the ideal spinel and the simulated surface.

\begin{tabular}{cccc}
\hline \hline & $\begin{array}{c}\text { Coordination } \\
\text { number }\end{array}$ & $\begin{array}{c}\text { Ideal } \\
\text { spinel }\end{array}$ & $\begin{array}{c}\text { Surface } \\
\text { structure }\end{array}$ \\
$\mathrm{Al}$ & 3 & 0.0 & 1.0 \\
& 4 & 28.1 & 56.4 \\
& 5 & 0.2 & 32.4 \\
& 6 & 71.7 & 10.1 \\
$\mathrm{O}$ & 2 & 0.6 & \\
& 3 & 38.1 & 16.7 \\
& 4 & 60.2 & 68.0 \\
& 5 & 1.4 & 13.9 \\
\hline \hline
\end{tabular}


nation numbers in general; therefore, three- and fivecoordinated aluminum atoms should be expected. However, three-coordinated aluminum ions are hardly found confirming their absence in experimental observations, ${ }^{3}$ and the number of five-coordinated cations is lower than expected if they were only due to the truncation of the bulk. This is due to the above-mentioned reconstruction process in which oxygens tend to emerge to the surface in order to saturate undercoordinated aluminum ions. This reconstruction process is also responsible for the formation of large cavities within the $\gamma-\mathrm{Al}_{2} \mathrm{O}_{3}$ structure. The relative proportion of octahedral plus five-coordinated to tetrahedral coordinated aluminum ions is close to that found in the ideal (110) surface plane because the system tends to expose similar planes both at the surface and within pore systems.

\section{CONCLUSIONS}

Molecular-dynamics simulations of the surface of $\gamma$ $\mathrm{Al}_{2} \mathrm{O}_{3}$ allows us to draw an alternative interpretation of its structure and to explain the roughness and porosity experimentally found for this material. Reconfiguration of surface ions gives way to pore formation with loss of long-range order. Both at the outer surface and at the pore surfaces, the exposed structures resemble the ideal (110) plane, as has been suggested from experimental work. Considering the surface as an amorphouslike phase, micropore volume and $\mathrm{x}$-ray-diffraction data can be well reproduced. Further work is being carried out in order to describe the reconstruction and cavity formation processes in a quantitative way.

\section{ACKNOWLEDGMENTS}

Financial support for this project was obtained from Comisión Interministerial de Ciencia y Tecnología (PB92-0665 and PB92-0662) of the Spanish Government and partially supported by Cray Research Inc. under supercomputing Grant No. DGAPASC-000192, UNAM (México). L.J.A. thanks the Junta de Andalucía for partially supporting a stay in the Universidad de Sevilla (AGCI-6006 and AGCI-1039).
*Author to whom correspondence should be addressed.

${ }^{1}$ J. B. Peri, J. Phys. Chem. 69, 220 (1965).

${ }^{2}$ B. C. Lippens and J. J. Steggerda, in Physical and Chemical Aspects of Adsorbents and Catalysts, edited by B. G. Linsen (Academic, New York, 1970), p. 171; A. Zecchina, S. Coluccia, and C. Morterra, Appl. Spectrosc. Rev. 21, 259 (1985).

${ }^{3}$ H. Knozinger and P. Ratnasamy, Catal. Rev. Sci. Eng. 17, 31 (1978).

${ }^{4}$ F. R. Chen, J. G. Davis, and J. J. Fripiat, J. Catal. 133, 263 (1992).

${ }^{5}$ L. J. Alvarez, J. Fernández Sanz, M. J. Capitán, and J. A. Odriozola, Chem. Phys. Lett. 192, 463 (1992).

${ }^{6}$ L. J. Alvarez, J. Fernández Sanz, M. J. Capitán, M. A. Centeno, and J. A. Odriozola, J. Chem. Soc. Faraday. Trans. 89, 3623 (1993).

${ }^{7}$ G. A. Somorjai, Chemistry in Two Dimensions (Cornell University Press, Ithaca, 1981).

${ }^{8}$ H. H. Kung, Stud. Surf. Sci. Catal. 45, 1 (1989); P. A. Thiel and T. E. Madey, Surf. Sci. Rep. 7, 211 (1987); S. Varma, G. S. Chottiner, and M. Arbab, J. Vac. Sci. Technol. A 10, 2857 (1992); C. C. Chang, J. Appl. Phys. 39, 5570 (1968); T. M. French and G. A. Somorjai, J. Phys. Chem. 74, 2489 (1970).
${ }^{9}$ D. J. Adams and I. R. McDonald, Physica B 79, 159 (1979).

${ }^{10}$ M. Dupuis, A. Farazdel, S. P. Karna, and S. A. Maluendes, in Modern Techniques in Computational Chemistry, edited by $\mathbf{E}$. Clementi (Escom, Leiden, The Netherlands, 1990), p. 277; M. Dupuis, S. Chin, and A. Márquez, in Relativistic and Electron Correlation Effects in Molecules and Solids, edited by G. L. Malli (Plenum, New York, 1994).

${ }^{11}$ R. D. Shannon and C. T. Prewitt, Acta Crystallogr. Sec. B 25, 925 (1969).

${ }^{12}$ L. J. Alvarez, L. E. León, and H. Muñoz (unpublished).

${ }^{13}$ SIMULA is a molecular dynamics and visualization software developed by L. J. Alvarez, Universidad Nacional Autónoma de México, 1993.

${ }^{14}$ S. Soled, J. Catal. 81, 252 (1983).

15J. P. Beaufils and Y. Barbaux, J. Chim. Phys. 78, 347 (1981); Y. Chen and L. Zhang, Catal. Lett. 12, 51 (1992).

${ }^{16} \gamma-\mathrm{Al}_{2} \mathrm{O}_{3}$ samples are obtained from Degussa (Aluminumoxid $C)$ labeled $\boldsymbol{A}$ in Fig. 7, and home prepared by precipitating with urea at $340 \mathrm{~K}$ an aqueous solution of aluminum nitrate. In this later case the precipitated was calcined at 1073, 1273, or $1473 \mathrm{~K}$ in air for $8 \mathrm{~h}$ prior to the obtainment of diffractograms $B, C$, and $D$, respectively, in Fig. 6 . 


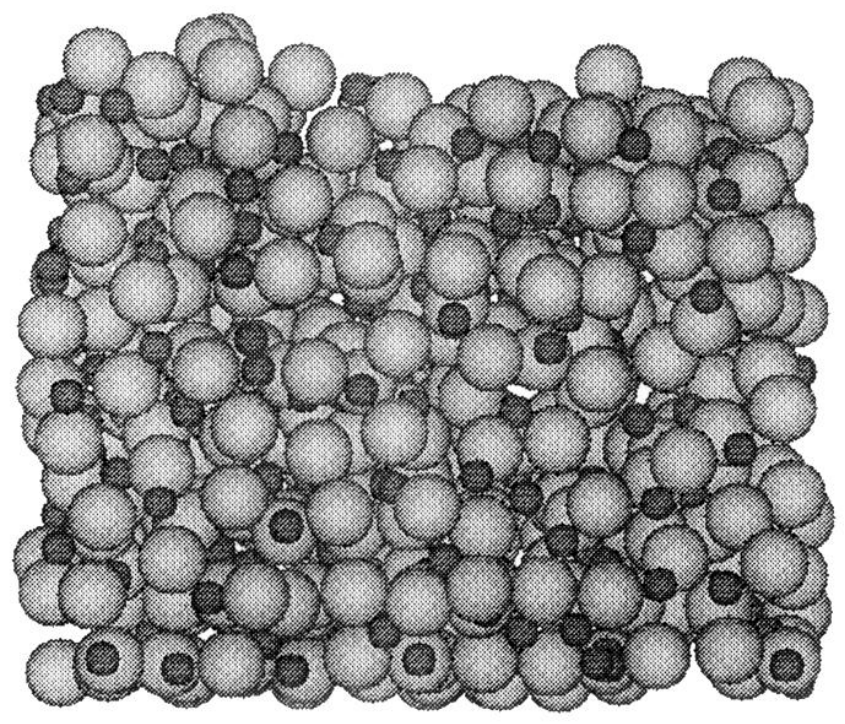

FIG. 1. Snapshot of the moving particles of the system after 15 ps simulation time. 